\title{
Choosing Wisely and Sharing Cost: A Multi-Bidding Cost Sharing Mechanism
}

\author{
Yoshio Kamijo ${ }^{1,2}$ \\ ${ }^{1}$ Department of Management, Kochi University of Technology, Kochi, Japan \\ ${ }^{2}$ Reseach Center for Social Design Engineering, Kochi University of Technology, Kochi, Japan \\ Email: yoshio.kamijo@gmail.com
}

Received 31 March 2014; revised 30 April 2014; accepted 24 May 2014

Copyright (C) 2014 by author and Scientific Research Publishing Inc. This work is licensed under the Creative Commons Attribution International License (CC BY). http://creativecommons.org/licenses/by/4.0/ cC) (i) Open Access

\begin{abstract}
We consider a situation where agents have to choose one project among the set of multiple alternatives and at the same time they have to agree with the way of sharing the cost of the project that is actually developed. We propose a multi-bidding cost sharing mechanism where each agent simultaneously announces his voluntary contribution for each project when the project is actually carried out, in combination with his vote for the projects. We show that a Nash equilibrium exists in this mechanism, and in any Nash equilibrium of this mechanism, the efficient project is always chosen. Moreover, in the Nash equilibrium, the way of sharing the cost of the project is, in a sense, an equal sharing rule.
\end{abstract}

\section{Keywords}

\section{Multi-Bidding Mechanism, Cost Sharing, Selection Rules}

\section{Introduction}

We consider a situation where agents have to choose one project among the set of multiple alternatives and at the same time they have to agree with the way of sharing the cost of the project that is actually developed. There are many examples of such situations from a location of public facility such as a public school, a disposal center, and a nuclear-related equipment to Joint venture development or R\&D investment agreement among firms.

In the pioneering work of [1], they formulated the mechanism that determines the project which will be developed and how the agents share the surplus from its development. In their mechanism called a multi-bidding mechanism, each individual is required to reveal payable bids for each project, which can be seen as his relative 
preference on the project, combined with the project announcement ${ }^{1}$. They show that their mechanism has a Nash equilibrium and always chooses the efficient projects. However, their mechanism is slightly artificial because the preference of each agent on the project is embedded in the announcement of his bids, which makes the agents in question to the announcement of his favorite project. Recently, [2] modifies the multi-bidding mechanism to a one where the project announcement is removed. He shows that in his natural mechanism, the existence of Nash equilibrium is no longer guaranteed and the desirability of the mechanism is limited.

The purpose of this paper is to extend their analysis one step forward. We consider a situation where there is a cost to develop the project and agents have to agree with how to share the cost of the project. In [1] and [2], the cost of the project is implicitly assumed to be zero or if it exists, agents are assumed to agree with the way of sharing the cost of project in advance of participating the mechanism. On the other hand, we consider how the agents share the cost of developing project, in addition to which project will be developed and how the agents share the surplus from its development. The introduction of the cost of projects needs a subtle rule of choosing a project because a project whose cost is not budgeted by the agents' payments cannot be carried out. In our mechanism, each agent is required to announce his voluntary contribution for each project when the project is actually carried out, in combination with his vote for the projects. A project is chosen from the set of projects whose cost is covered by the agents' voluntary contributions. From such the set of projects, one project is more likely to be chosen if it is voted by more agents. When there is no project in the set that is voted, one project is chosen from the set of projects whose cost is strictly covered by the agents' voluntary contribution. We show that our mechanism has a Nash equilibrium, and in any Nash equilibrium of our mechanism, an efficient project is chosen and the cost of its development is exactly covered by the agents' voluntary contributions.

The reminder of the paper is as follows. In the next section, we present our mechanism, a multi-bidding cost sharing mechanism, and then, we examine the properties of the mechanism. In Section 3, we conclude the paper with some discussion on the relation of our results with the ones obtained from previous studies.

\section{A Multi-Bidding Cost Sharing Mechanism}

\subsection{Setting}

We consider a set of agents $N=\{1, \cdots, n\}$ and a set of possible projects $K=\{1, \cdots, k\}$ with $n \geq 2$ and $k \geq 2$. The utility of agent $i$ that is directly obtained from project $q$ is $v_{q}^{i}$. The cost of developing project $q$ is $c_{q}$, $c_{q}>0$, which must be covered by the agents if project $q$ is actually carried out ${ }^{2}$. The aggregate cost of all the projects is denoted by $C=\sum_{q \in K} c_{q}$. Project $q$ is efficient if

$$
\sum_{i \in N} v_{q}^{i}-c_{q} \geq \sum_{i \in N} v_{q^{\prime}}^{i}-c_{q^{\prime}}
$$

for all $q^{\prime} \in K$. We denote by $E$ the set of efficient projects. Because the set of projects is finite, $E$ is not an empty set. We denote by $V^{e}$ the maximum total pure value that can be obtained by developing the project, that is,

$$
V^{e}=\sum_{i \in N} v_{q}^{i}-c_{q}
$$

for some $q \in E$.

One benchmark mechanism that determines the project that is developed and how the cost of the project is shared is a mechanism that randomly chooses a project and shares the cost of the project equally among the agents. In this random selection and equal sharing mechanism, the expected payoff of agent $i$ is

$$
\underline{v}_{i}=\frac{1}{k} \sum_{q \in K}\left(v_{q}^{i}-\frac{c_{q}}{n}\right) \text {. }
$$

\footnotetext{
${ }^{1}$ Mechanisms based on individuals' bids have been intensively explored in the recent literature on the implementation of a solution concept established in cooperative game theory. Reference [3] formulated the mechanism involving bidding stage where each individual is required to reveal payable bids to each of the other individuals in exchange for becoming a proposer in the subsequent stages, and they showed that in its subgame perfect equilibrium the Shapley value is realized as a final payoff to each individual. This approach is applied for the implementation of the variants of the Shapley value [4]-[7]. In other contexts, [8] apply the mechanism in a local public goods economy, and [9] apply it in a network formation game.

${ }^{2}$ References [1] and [2] consider the case where $c_{q}$ is zero for all the projects. This can be seen as the situation where a project includes the way of sharing cost of the project. In other words, in their models, the agents have already agreed with the way of sharing cost, in a sense.
} 
This simple mechanism has several deficiencies. This mechanism may choose an inefficient project, which means that after the determination of the project by the mechanism, agents may start to bargain because there is a room for all of them to be better off by selecting an efficient project, and this diminishes the credibility of the mechanism. Second, it is uncertain that equal sharing of the cost of project is an appropriate way of sharing the cost among agents when the agents' preferences show the diversity. The mechanism a priori assumes the desirability of the equal sharing. These points motivate to consider another mechanism that chooses the project and determines the way of sharing the costs of projects.

\subsection{Mechanism Design}

The multi-bidding mechanism of [1] determines the project which will be developed and how the agents share the surplus from its development. Their mechanism proceeds as follows: each agent announces $k$ bids, one for each possible project, that are constrained to sum up to zero. In addition, agent $i$ announces a project that he wants to be developed. The project with the highest aggregate bid is chosen as the winner. In case there is a tie, the winning project is randomly chosen among those with the highest aggregate bid and which are announced by at least one agent.

Reference [2] considers that the multi-bidding mechanism of [1] is slightly artificial because on the one hand, each agent announces his bids to any possible project, which may be seen as some measure of his preference on the projects, and on the other hand, each agent has to announce a project. Reference [2] proposes a natural multibidding mechanism where each agent announces the bids for each possible project and there is no project announcement. In Ehlers's mechanism, the project with highest net bid that are placed on the maximum bid by at least one agent is chosen by the mechanism.

The problem considered in this paper involves the stage immediately prior to [1] and [2]. In other words, we consider further additional problem that are not sufficiently discussed by their work. Thus, we consider how the agents share the cost of developing project, in addition to which project will be developed and how the agents share the surplus from its development. Moreover, the introduction of the cost of projects needs a subtle rule of choosing a project because a project whose cost is not covered by the agents cannot be carried out.

We also consider the natural version of the multi-bidding mechanism in a different way from [2], in addition to dealing with the cost sharing problem. The Ehlers's critique on the multi-bidding mechanism is why agents should doubly announce his preference on the projects, one embedded in his bid profile and the other represented by the project announcement. Thus, [2] removes the project announcement from his mechanism. Here, we take another root to resolve the unnaturalness of the multi-bidding mechanism; in our mechanism, the bid for each project made by an agent has a natural interpretation different from the measurement of agent's preference on the projects.

Our mechanism is informally described as follows. Similar to the multi-bidding mechanism, each agent announces $k$ bids, one for each possible project, which is interpreted as his cost burden for the development of the project if it is actually chosen. In addition, agent $i$ votes on the projects. Only the projects whose cost is collected by agents will be chosen as the winner, and among such projects, the project that is voted by more agents is chosen at a higher probability.

A multi-bidding cost sharing (MBCS) mechanism is described as follows. Each $i$ announces $k$ bids, one for each possible project, that are constrained to sum up to per capita total cost $\bar{c}$, that is defined by $\bar{c}=\frac{1}{n} C=\frac{1}{n} \sum_{q \in K} c_{q}$. Thus, $i$ chooses $k$-dimensional vector $b^{i}=\left(b_{q}^{i}\right)_{q \in K}$ such that $\sum_{q \in K} b_{q}^{i}=\bar{c}$. In addition, $i$ votes on projects that he wants to be chosen as a winner. Here, $i$ is allowed to vote for all the projects that he likes. Thus, his strategy is a vector $\left(b^{i}, M^{i}\right) \in H \times 2^{K} \backslash \varnothing$, where

$$
H=\left\{b \in \mathbb{R}^{K}: \sum_{q \in K} b_{q}=\bar{c}\right\} .
$$

The strategy profile of $n$ agents is $\mathbf{S}=(b, \mathbf{M})$ where $b=\left(b^{i}\right)_{i \in N}$ is a bid profile and $\mathbf{M}=\left(M_{i}\right)_{i \in N}$ is a vote profile. Given an announced bid profile $b$, an aggregate bid of project $q$ is $B_{q}=\sum_{i \in N} b_{q}^{i}$, and an aggregate net bid of project $q$ is $\bar{B}_{q}=B_{q}-c_{q}$. The set of project whose aggregate net bid is non-negative is denoted by $\Omega_{0}(b)=\left\{q \in K: \bar{B}_{q} \geq 0\right\}$. Let $d_{q}(\mathbf{M})$ be the number of agents that vote for project $q$, that is, 
$d_{q}(\mathbf{M})=\left|\left\{i \in N: q \in M^{i}\right\}\right|$. The score of project $q$ is $s_{q}(b, \mathbf{M})$ which is defined by

$$
s_{q}(b, \mathbf{M})= \begin{cases}d_{q}(\mathbf{M})+\eta & \text { if } \bar{B}_{q}>0 \\ d_{q}(\mathbf{M}) & \text { if } \bar{B}_{q}=0,\end{cases}
$$

where $\eta, 0<\eta<1$, can be seen as the little bonus for the project that has positive aggregate net bid $^{3}$. A winner of the mechanism is chosen from $\Omega_{0}(b)$. If there are multiple members in $\Omega_{0}(b)$, a project is chosen in a probability proportional to its score. One remark on our selection procedure is that because of the constrain on the bids, $\Omega_{0}(b)$ is always non-empty, which can be confirmed by

$$
\sum_{q \in K} \bar{B}_{q}=\sum_{i \in N} \sum_{q \in K} b_{q}^{i}-\sum_{q \in K} c_{q}=n \bar{c}-C=0 .
$$

Moreover, there is no case that all the members of $\Omega_{0}(b)$ have zero score because if $\Omega_{0}(b) \neq K$, there must exist some project $q \in \Omega_{0}(b)$ whose aggregate net bid is positive, and if $\Omega_{0}(b)=K$, there must exist some project $q \in \Omega_{0}(b)$ that is voted by at least one agent.

If the mechanism chooses project $q$, then $q$ is developed and each $i$ receives the payments $\frac{1}{n} \bar{B}_{q}-b_{q}^{i}$. Agent $i$ 's payoff is thus $v_{q}^{i}+\frac{1}{n} \bar{B}_{q}-b_{q}^{i}$. Note that according to our mechanism, $\bar{B}_{q}$ for the winning project $q$ is always non-negative, and thus, $\frac{1}{n} \bar{B}_{q}$ is seen as the return of excessive amount of money collected for developing the project $^{4,5}$.

\section{Results}

We use a Nash equilibrium as our equilibrium concept to analyze the mechanism.

The next lemma shows that the aggregate net bid is zero for any project at a Nash equilibrium.

Lemma 1. In any Nash equilibrium of the MBCS mechanism, the aggregate net bid of projects is zero. That is, $\bar{B}_{q}=0$ at any Nash equilibrium for any $q \in K$.

Proof. When $\Omega_{0}(b)=K$, we have the desired result because of Equation (1). Thus we consider the case $\Omega_{0}(b) \neq K$. Then, this implies that there exists some $p \in \Omega_{0}(b)$ such that $\bar{B}_{q}>0$. Because of the construction of the score function, project $p$ can be chosen by the mechanism in a positive probability. Therefore, agent $i$ can improve his payoff by reducing $b_{q}^{i}$ by small $\epsilon$ and increasing the bid for another project in $K \backslash \Omega_{0}(b)$ by $\epsilon$ when $\epsilon$ is small enough. This is a contradiction. Q.E.D

From Lemma 1, we know that a project may be chosen by some probability at a Nash equilibrium. The next lemma show that the project that is chosen in a positive probability at a Nash equilibrium gives the highest payoff to any agent given the Nash equilibrium bid profile $b$.

Lemma 2. If project $q$ is chosen by the mechanism at Nash equilibrium $\mathbf{S}=(b, \mathbf{M})$ in a positive probability, this $q$ must satisfy

$$
v_{q}^{i}-b_{q}^{i} \geq v_{p}^{i}-b_{p}^{i} \text { for any } i \in N \text { and for any } p \in K \text {. }
$$

Proof. We prove this by the way of contradiction. We assume that there exist agent $i$ and project $p \in \Omega_{0}(b)=K$ such that

$$
v_{q}^{i}-b_{q}^{i}<v_{p}^{i}-b_{p}^{i} .
$$

\footnotetext{
${ }^{3}$ This asymmetry treatment between projects with positive aggregate net bid and ones with balanced aggregate net bid is needed to avoid the case that the score of all the members in $\Omega_{0}(b)$ is zero.

${ }^{4}$ Similar to [1] and [2], our results do not depend on the equal sharing distribution of the excessive amount of money.

${ }^{5}$ Because the selection procedure of the MBCS mechanism is different from that of the multi-bidding mechanism by [1] and that of the natural multi-bidding mechanism by [2], our mechanism does not coincide with their mechanisms even when $c_{q}=0$ for all $q \in K$. However as we will show in the next subsection, all the desirable properties of the multi bidding mechanism are satisfied by our mechanism.
} 
Let $\hat{p}$ be the maximizer of $v_{p}^{i}-b_{p}^{i}$ among the set of projects. Then, agent $i$ can improve his payoff by reducing $b_{q}^{i}$ by small $\epsilon$ and increasing the bid for project $\hat{p}$ by $\epsilon$ when $\epsilon$ is small enough. This contradicts the Nash equilibrium. Q.E.D

The following theorem guarantees the efficiency of the projects that are chosen by the MBCS mechanism.

Theorem 1. If project $q$ is chosen by the mechanism at Nash equilibrium at a positive probability, this project is efficient.

Proof. Let $q$ denote the project with a positive score. By summing the condition of Lemma 2 over $i \in N$, we have

$$
\sum_{i \in N} v_{q}^{i}-\sum_{i \in N} b_{q}^{i} \geq \sum_{i \in N} v_{p}^{i}-\sum_{i \in N} b_{p}^{i}
$$

By Lemma 1, this can be reduced to

$$
\sum_{i \in N} v_{q}^{i}-c_{q} \geq \sum_{i \in N} v_{p}^{i}-c_{p}
$$

Thus, $q$ is efficient. Q.E.D

The next theorem shows that the lower bound of the agent's utility when they participate in the MBCS mechanism is $\underline{v}^{i}$, the utility of agent $i$ when the cost of the projects are shared by the equal sharing rule and all of the projects are chosen in an equal probability:

Theorem 2. In any Nash equilibrium, the final payoff of agent $i$ is greater than or equal to $\underline{v}^{i}$.

Proof. Let $q$ be the project that has a positive score. By summing the condition in Lemma 2 over the set of projects, we have

$$
k\left(v_{q}^{i}-b_{q}^{i}\right) \geq \sum_{p \in K}\left(v_{p}^{i}-b_{p}^{i}\right)
$$

By the definition of the mechanism, $\sum_{p \in K} b_{p}^{i}=\bar{c}=\frac{1}{n} \sum_{p \in K} c_{p}$. Thus, the left hand side of the above equation is

$$
\sum_{p \in K}\left(v_{p}^{i}-\frac{c_{p}}{n}\right)
$$

Divide the equation by $k$, and we have the desired result. Q.E.D

The following lemma describes the Nash equilibrium vote profile.

Lemma 3. In any Nash equilibrium, for any $i \in N, M_{i} \subseteq E$.

Proof. Let $q$ be an inefficient project. When $q \in M_{i}$ for some $i$, Lemma 1 means that this inefficient $q$ will be chosen by the mechanism at a positive probability. This contradicts Theorem 1. Q.E.D

The following lemma shows that the indifference between projects $p$ and $q$ which are possibly chosen by the mechanism for any $i \in N$ at Nash equilibrium.

Lemma 4. In any Nash equilibrium, for any $i \in N$, the final payoff of agent $i$ is indifferent between any two projects that can be chosen by the mechanism.

Proof. Let $p$ and $q$ be projects with a positive score at Nash equilibrium. The condition of Lemma 2 must be satisfied in equality for any agent. Q.E.D

From the above lemma, we have the important properties of Nash equilibrium of the MBCS mechanism. Even if there are two efficient projects that can be chosen by the mechanism at a Nash equilibrium, the final payoff of each $i$ is indifferent between these two projects. 
The following lemma characterizes the Nash equilibrium.

Lemma 5. A strategy profile becomes a Nash equilibrium if and only if the following three conditions are satisfied:

1) $\bar{B}_{q}=0$ for any $q \in K$,

2) Given a bids profile, the final payoff of each $i$ is maximized when an efficient project is chosen, and it is indifferent between any two projects that are efficient, and

3) $M_{i} \subseteq E$ for each $i \in N$.

Proof. We have already shows the "only if part". So we will show the "if part" of the theorem. However, the proof of the "if part" is almost same as the proof of Lemma 5 in [1]. Thus, we omit it. Q.E.D

Let us define the set of payoffs $P$ as follows:

$$
P=\left\{u=\left(u^{1}, \cdots, u^{n}\right) \in \mathbb{R}^{N}: \sum_{i \in N} u^{i}=V^{e} \text { and } u^{i} \geq \underline{v}^{i}\right\} .
$$

Theorem 3. Any payoff vector $u$ in $P$ can be a Nash equilibrium payoff of the MBCS mechanism.

Proof. The proof of this theorem is similar to the proof of Theorem 3 in [1]. Thus, we only explain the outline of the proof and give a small remark on the different point from their proof.

This theorem is proved by the following manner. First, we show that the set of Nash equilibrium and the set of Nash equilibrium payoffs are convex. Then, we show that for any agent $i$ there exists a Nash equilibrium such that all the agents other than $i$ obtain the payoffs of their lower bound, i.e., $\underline{v}^{j}$. Finally, we end the proof by showing that these payoff vectors are the end points of $P$.

The different point from the proof of [1] is a bid profile that corresponds to the end point of the set of Nash equilibrium profiles. Take any agent $j \in N$. We consider the following strategy that can be shown to the best for agent $j$ among the set of Nash equilibria:

$$
\begin{gathered}
b_{q}^{i}=v_{q}^{i}-\underline{v}^{i} \text { for each } i \neq j, q \in K, \\
b_{q}^{j}=\sum_{i \neq j} \underline{v}^{i}-\sum_{i \neq j} v_{q}^{i}+c_{q} \text { for each } q \in K .
\end{gathered}
$$

Each agent's strategy consists of the above bids profiles and the vote set that is a subset of $E$. Then, it is shown that the above strategy profile is a Nash equilibrium and becomes the end point of the set of Nash equilibrium bid profiles. Q.E.D

The next theorem says that any Nash equilibrium of the MBCS mechanism is a strong Nash equilibrium.

Theorem 4. Any Nash equilibrium of the MBCS mechanism is a strong Nash equilibrium of the mechanism.

Proof. The proof of this theorem is almost same as the proof of Theorem 4 in [1]. Thus, we omit it. Q.E.D

\section{Discussion}

An important implication obtained from our analysis is that the set of final payoffs that can be supported by Nash equilibrium (or strong Nash equilibrium) of the MBCS mechanism is the same as the set of payoffs that are supported by (strong) Nash equilibrium of the multi-bidding mechanism applied to the situation where the utility obtained from project is transformed to $\tilde{v}_{q}^{i}=v_{q}^{i}-\frac{c_{q}}{n}$. This means that if agents can agree with the equal sharing rule of the cost of the project in advance of playing the multi-bidding mechanism, they can reach the same result as the one in the MBCS mechanism. However, it is uncertain that such an agreement can be formed before the mechanism, and in that case, the MBCS mechanism will be functioned.

What our mechanism leads to if all the projects are efficient should be emphasized. From Theorem 2, we know that in any Nash equilibrium, all the agents obtain the utility greater than or equal to their lower bound, $\underline{v}_{i}$, and under the circumstance where $K=E$, the summation of their lower bound utilities must be $V^{e}$. This implies that when $K=E$, the Nash equilibrium payoff is uniquely determined, i.e., $\left(\underline{v}_{1}, \cdots, \underline{v}_{n}\right)$. Thus, our 
mechanism leads to the utility profile which is the same as the expected utility in the random selection and equal sharing mechanism where each project is chosen at an equal probability and the cost of chosen project is shared equally among agents. However, it should be noticed that as in the case with other bidding-based mechanisms, our mechanism achieves the payoff vector $\left(\underline{v}_{1}, \cdots, \underline{v}_{n}\right)$ as a realized value but not an expected value.

One way to extend our mechanism to the asymmetric case is that burden share of each agent is determined by some exogenous factors but there is some difficulty to reach an agreement on this burden share. In such a case, if we apply the modified version of multi-bidding cost sharing mechanism where now the bids of agent $i$ is constrained to sum up $\sum_{q \in K} t_{i}^{q} c^{q}$, where $t_{i}^{q}$ is normalized burden share of agent $i$ to project $q$, this mechanism always selects an efficient project at every Nash and strong Nash equilibrium. The difference from the previous mechanism is that in this modified mechanism, the lower bound utility of agent $i$ at Nash equilibrium is

$\underline{\underline{v}}_{i}=\frac{1}{k} \sum_{q \in K}\left(v_{i}^{q}-t_{i}^{q} c_{q}\right)$.

\section{Acknowledgements}

The author thanks Kohei Kamaga for their helpful comments and suggestions.

\section{References}

[1] Perez-Castrillo, D. and Wettstein, D. (2002) Choosing Wisely: A Multi-Bidding Approach. American Economic Review, 92, 1577-1587. http://dx.doi.org/10.1257/000282802762024647

[2] Ehlers, L. (2009) Choosing Wisely: The Natural Multi-Bidding Mechanism. Economic Theory, 39, 505-512. http://dx.doi.org/10.1007/s00199-008-0355-7

[3] Perez-Castrillo, D. and Wettstein, D. (2001) Bidding for the Surplus: A Non-Cooperative Approach to the Shapley Value. Journal of Economic Theory, 100, 274-294. http://dx.doi.org/10.1006/jeth.2000.2704

[4] Vidal-Puga, J. and Bergantinos, G. (2003) An Implementation of the Owen Value. Games and Economic Behavior, 44, 412-427. http://dx.doi.org/10.1016/S0899-8256(03)00043-5

[5] Kamijo, Y. (2008) Implementation of Weighted Values in Hierarchical and Horizontal Cooperation Structure. Mathematical Social Sciences, 56, 336-349. http://dx.doi.org/10.1016/j.mathsocsci.2008.06.002

[6] Slikker, M. (2007) Bidding for Surplus in Network Allocation Problems. Journal of Economic Theory, 137, $493-511$. http://dx.doi.org/10.1016/j.jet.2007.01.010

[7] Ju, Y. and Wettstein, D. (2009) Implementing Cooperative Solution Concepts: A Generalized Bidding Approach. Economic Theory, 39, 306-330. http://dx.doi.org/10.1007/s00199-008-0335-y

[8] Mutuswami, S., Perez-Castrillo, D. and Wettstein, D. (2004) Bidding for the Surplus: Realizing Efficient Outcomes in Economic Environments. Games and Economic Behavior, 48, 111-123. http://dx.doi.org/10.1016/j.geb.2003.08.003

[9] Perez-Castrillo, D. and Wettstein, D. (2005) Forming Efficient Networks. Economics Letters, 87, 83-87. http://dx.doi.org/10.1016/j.econlet.2004.10.005 